\title{
Selective uptake and refolding of globular proteins in coacervate micro-droplets
}

\author{
Nicolas Martin, Mei Li and Stephen Mann*
}

Centre for Protolife Research and Centre for Organized Matter Chemistry, School of Chemistry, University of Bristol, Bristol BS8 1TS, UK

*s.mann@bristol.ac.uk

\section{Supplementary Information}

\section{Experimental Methods:}

1. Materials

2. Fluorescent labelling of protein

3. Fluorescent labelling of PAA

4. Zeta potential measurements

5. Turbidimetry

6. Determination of coacervate composition, water and urea content, density and refractive index

\section{SI Figures}

1. Urea-dependent change in the equilibrium partition coefficient of FITC-labelled proteins (BSA, CAB) and uncoupled fluorescein

2. Sequestration and spatial distribution of CAB-FITC and $\alpha$-ChT-FITC in PDDA/PAA coacervate micro-droplets at increasing urea concentrations

3. Time-dependent exponential decrease of the mean fluorescence intensity inside individual coacervate micro-droplets after addition of various urea concentrations

4. Far-UV SRCD spectra showing denaturation of BSA in the PDDA/PAA bulk coacervate phase at increasing urea concentrations

5. Turbidity and zeta potential of PDDA/PAA coacervate droplet dispersions in water/urea mixtures

6. Decrease in turbidity for coacervates prepared from mixtures of PDDA and BSA with increasing urea concentration

7. Physico-chemical characterization of coacervate composition, water and urea content, density and refractive index

8. Sequestration and spatial distribution of BSA-FITC and CAB-FITC PDDA/PAA coacervate micro-droplets at different droplets surface charge

9. Spatial distribution of CAB-FITC in negatively charged PDDA/PAA coacervate droplets at increasing urea concentration 


\section{Materials}

Poly(sodium acrylate) (PAA) and poly(diallyldimethylammonium chloride) (PDDA) with chain lengths of ca. 50 monomers $\left(5,100 \mathrm{~g} \mathrm{~mol}^{-1}\right.$ (PAA); 8,500 $\mathrm{g} \mathrm{mol}^{-1}$ (PDDA)), bovine serum albumin (BSA), carbonic anhydrase from bovine erythrocytes (CAB) and $\alpha$-chymotrypsin from bovine pancreas $(\alpha-C h T)$ were used as received. Anhydrous $N$-methylpyrrolidone (NMP), triethylamine (TEA), $N, N^{\prime}$-dicyclohexylcarbodiimide (DCC), sodium methoxide (25 wt\% in methanol), fluorescein amine (isomer II) and fluorescein isothiocyanate (FITC) were used for the fluorescent labelling of polyelectrolytes and proteins. Urea, trizma-base and $1 \mathrm{M}$ solutions of hydrochloric acid and sodium hydroxide were used as buffers, and 3[methoxy(polyethyleneoxy)propyl]trimethoxysilane (ABCR GmbH) was used to functionalize the surface of microscope capillary slides. Milli-Q water $\left(18.2 \mathrm{M} \Omega . \mathrm{cm}^{-1}\right)$ was used for all experiments, and unless otherwise specified, all chemicals and biomolecules were purchased from Sigma-Aldrich.

\section{Fluorescent labelling of proteins}

Protein solutions ( $4 \mathrm{mg} \mathrm{mL}^{-1}$ ) were prepared by dissolving freeze-dried protein powders in 0.5 $\mathrm{M} \mathrm{NaHCO}_{3} / \mathrm{NaCO}_{3}$ buffer at $\mathrm{pH}$ 9.5. An aliquot of a freshly-prepared anhydrous DMSO solution of fluorescein isothiocyanate (FITC) $\left(10 \mathrm{mg} \mathrm{mL}^{-1}\right)$ was added to the protein solution at a FITC : protein final molar ratio of ca. $20: 1$. The reaction mixture was kept at room temperature in the dark for 2-4 hours, then purified by size exclusion chromatography using a Sephadex G-25 resin (Sigma-Aldrich) eluted with $10 \mathrm{mM}$ Tris-HCl buffer $\mathrm{pH}$ 8. The concentration of the FITC-labelled protein in the collected fractions was determined by UV-visible spectrophotometry according to the relationship: [protein] $=\left(A_{280}-0.33 \times A_{495}\right) / \varepsilon_{\text {protein, }}$ where $A_{280}$ and $A_{495}$ were the absorbances at 280 and $495 \mathrm{~nm}, 0.33$ the correction factor to account for the dye absorption at $280 \mathrm{~nm}$, and $\varepsilon_{\text {protein }}$ the extinction coefficient of the protein $\left(0.665 \mathrm{~mL} \mathrm{mg}^{-1} \mathrm{~cm}^{-1}\right.$ (BSA) and 1.83 $\left.\mathrm{mL} \mathrm{mg} \mathrm{cm}^{-1}(C A B)\right)$. The FITC : protein final molar ratio was determined from the ratio $\left(\mathrm{A}_{495} /\right.$

$\left.\varepsilon_{\text {FITC }}\right) /\left(\left[\right.\right.$ protein] $\left(\mathrm{mg} \mathrm{mL}^{-1}\right) / \mathrm{M}_{\text {protein }}$ ), where $\varepsilon_{\text {FITC }}$ was the molar extinction coefficient of FITC at $495 \mathrm{~nm}\left(69,000 \mathrm{~mol}^{-1} \mathrm{~L} \mathrm{~cm}^{-1}\right)$, and $M_{\text {protein }}$ the molar mass of the protein $\left(66,500 \mathrm{~g} \mathrm{~mol}^{-1}\right.$ (BSA) and $\left.29,000 \mathrm{~g} \mathrm{~mol}^{-1}(\mathrm{CAB})\right)$. Typically, the value FITC : protein was ca. $4: 1$.

\section{Fluorescent labelling of PAA}

Poly(acrylic acid) was labelled with fluorescein following a published procedure [D. F. Anghel, et al., Polymer 1998, 39, 3035-3044; E. Spruijt, et al., Macromolecules 2010, 43, 6476-6484]. Briefly, $0.35 \mathrm{~g}$ of PAA (acidic form) was dissolved in $7 \mathrm{~mL}$ of $\mathrm{N}$-methylpyrrolidone at $60^{\circ} \mathrm{C}$. After ca. $1 \mathrm{~h}$, a mixture containing $7.5 \mu \mathrm{L}$ triethylamine (TEA) (0.02 equiv. compared to acrylic acid monomers) and $11 \mathrm{mg}$ of $N, N^{\prime}$-dicyclohexylcarbodiimide (DCC; 0.02 equiv.) dissolved in $0.5 \mathrm{~mL}$ anhydrous $N$-methylpyrrolidone (NMP) was added to the PAA solution, followed by addition of $19.6 \mathrm{mg}$ of fluorescein amine (0.021 equiv.) in $0.1 \mathrm{~mL}$ DMSO. The resulting clear, pale yellow mixture was stirred in the dark at $60^{\circ} \mathrm{C}$ for $24 \mathrm{~h}$. After cooling to room temperature, PAA was precipitated by dropwise addition of a 25 wt\% sodium methoxide in methanol solution ( 2 equiv.). The orange precipitate was collected by centrifugation (8,000 rpm, $20 \mathrm{~min})$, redissolved in a minimum amount of water and precipitated in methanol. This procedure was repeated three times. The final precipitate was dissolved in water and freeze-dried, yielding the sodium salt of fluorescein-labelled PAA as a pale yellow powder. The labelling efficiency was 
determined by dissolving a known amount of the labelled polymer in water, adjusting the $\mathrm{pH}$ to 9, and recording the UV-visible absorption spectrum. The molar extinction coefficient of fluorescein amine at $490 \mathrm{~nm}$ was determined as $76,000 \mathrm{~mol}^{-1} \mathrm{~L} \mathrm{~cm}^{-1}$ at $\mathrm{pH}$ 9. The labelling ratio was 1 : 14800, equivalent on average to the labelling of 1 per 270 polymer chains; thus, the probability of more than one fluorescein label per polymer was low and was not expected to influence the phase behaviour of the labelled-PAA/PDDA mixtures.

\section{Zeta potential measurements}

Zeta potential measurements were recorded at room temperature on PDDA/PAA coacervate micro-droplets prepared in $10 \mathrm{mM}$ Tris- $\mathrm{HCl}(\mathrm{pH} \mathrm{8)}$ at different PDDA : PAA monomer ratios (0.55-1.4) with a fixed final PDDA monomer concentration of $1 \mathrm{mM}$. Samples were injected into a disposable folded capillary cuvette and analysed using a Malvern Zetasizer Nano ZS instrument (Malvern Instruments). The average value and standard deviation of three successive measurements were determined.

\section{Turbidimetry}

Turbidity was used to qualitatively assess the extent of coacervation as a function of urea concentration. Measurements were performed at $500 \mathrm{~nm}$ and $25^{\circ} \mathrm{C}$ using a plate reader equipped with a UV-vis spectrophotometer (CLARIOstar, BMG LabTech).

\section{Determination of coacervate composition, water and urea content, density and refractive index}

Stock solutions of PAA and PDDA were prepared at a monomer concentration of $640 \mathrm{mM}(60 \mathrm{~g}$ $\mathrm{L}^{-1}$ (PAA); $103 \mathrm{~g} \mathrm{~L}^{-1}$ (PDDA)) in $10 \mathrm{mM}$ Tris- $\mathrm{HCl}$ buffer, $\mathrm{pH}$ 8, in the absence or presence of $10 \mathrm{M}$ urea. A PDDA/PAA coacervate was prepared from an equimolar mixture of the oppositely charged polyelectrolytes ( $5 \mathrm{~mL}, 80 \mathrm{mM}$ final monomer concentration) by mixing aliquots of the polymer stock solutions in $10 \mathrm{mM}$ Tris- $\mathrm{HCl}$ buffer at $\mathrm{pH} 8$ and supplemented with appropriate volumes of a $10 \mathrm{M}$ urea stock solution to give final urea concentrations between 0 and $10 \mathrm{M}$ (with $1 \mathrm{M}$ increments). The coacervate suspensions were shaken vigorously and then left to equilibrate for 2 days at room temperature, after which a clear polyelectrolyte-rich phase (coacervate) sedimented. Centrifugation (5,000 rpm, $10 \mathrm{~min}$ ) ensured complete phase separation between the denser coacervate phase and the polymer-poor supernatant. A given volume of the sedimented coacervate phase was weighed to determine its density, and then dried in an oven at $110^{\circ} \mathrm{C}$ until the dry weight remained constant. The water content of the coacervate was calculated using the weight difference before and after drying.

To determine the polyelectrolyte composition of the coacervate, $50 \%$ of the PAA used for PDDA/PAA coacervation was replaced by FITC-labelled PAA. The concentrations of FITC-PAA in the two phases produced by centrifugation were measured by UV-vis spectrometry $\left(\lambda_{\max }=\right.$ $490 \mathrm{~nm}$ ) after adjustment of the supernatant $\mathrm{pH}$ to 9 by dropwise addition of a $1 \mathrm{M} \mathrm{NaOH}$ solution, or after dilution of an aliquot of the coacervate phase into $0.5 \mathrm{M} \mathrm{NaCl}$ at pH 9 to disassemble the PDDA/PAA complex. The AA monomer concentration was determined from both phases using the determined labelling ratio of the FITC to PAA chains (1 : 14800 molar ratio; 1 per 270 polymer chains), the mixing ratio of labelled and unlabelled PAA (1:1), and the concentration of the fluorescein dye in both phases. 
The refractive indices of the supernatant and the coacervate phase were measured at different urea concentrations using a refractometer (Bellingham \& Stanley, London). The values were compared to the refractive indices of pure urea and pure polymer solutions, and the differences used to estimate the distribution of urea between the coacervate and supernatant. The concentrations of urea in the coacervate and supernatant were also estimated from the dry weight of the coacervate phase and the measured content of PAA, assuming a constant PDDA/PAA molar ratio for coacervates prepared in different concentrations of urea. Both methods gave similar estimations of the urea content. 

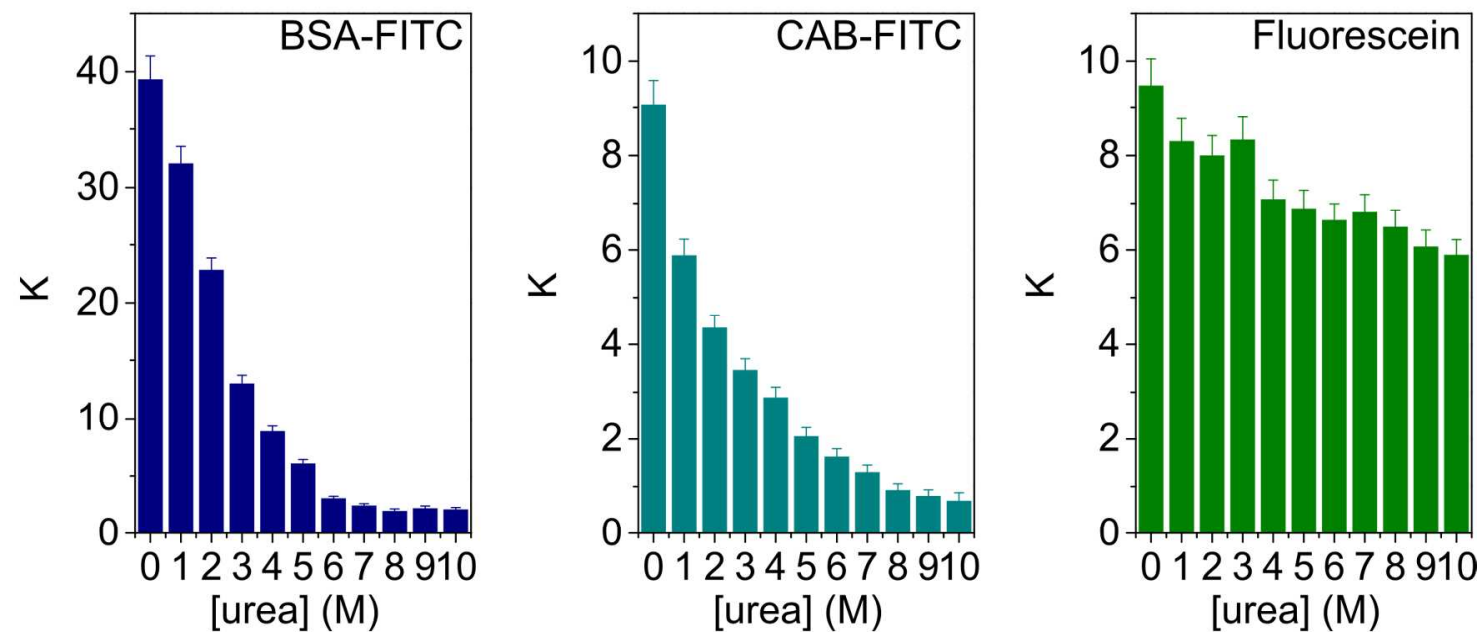

Figure S1. Plots showing urea-dependent change in the equilibrium partition coefficient (K) of FITC-labelled proteins (BSA, CAB), and uncoupled fluorescein. 

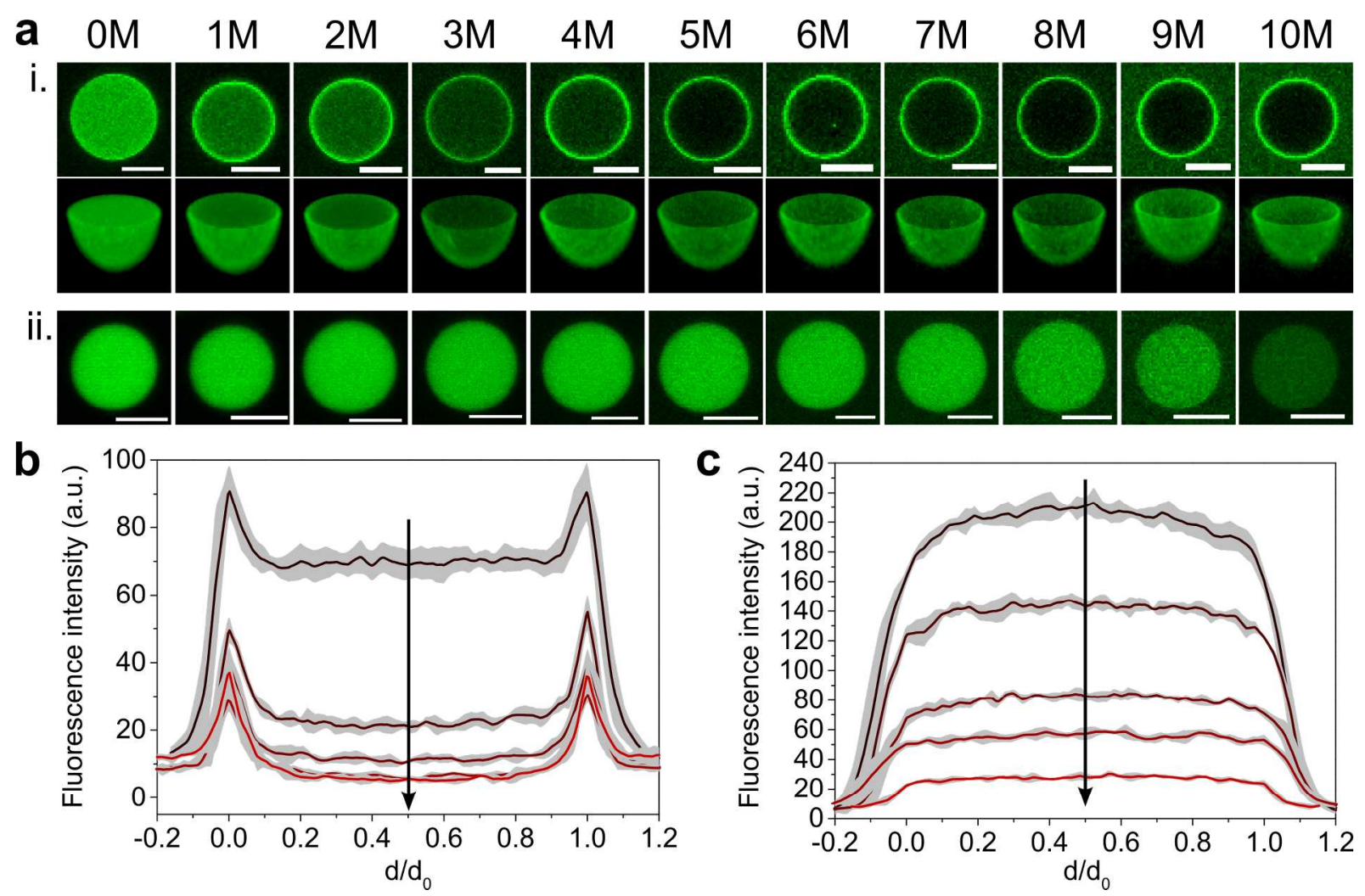

Figure S2. (a) (i) Confocal fluorescence microscopy images and 3D reconstructions of single CAB-FITC-containing PDDA/PAA coacervate droplets $(\mathrm{pH} 8$; final monomer concentration, 25 $\mathrm{mM}$; monomer molar ratio $=1: 1$ ) prepared at urea concentrations between 0 and $10 \mathrm{M}$ showing changes in the spatial distribution of $C A B$ during protein unfolding; scale bars $5 \mu \mathrm{m}$. (ii) Confocal fluorescence microscopy images of single $\alpha$-ChT-FITC-containing PDDA/PAA coacervate droplets prepared at urea concentrations between 0 and $10 \mathrm{M}$; scale bars $5 \mu \mathrm{m}$. $(\mathbf{b}, \mathbf{c})$ Fluorescence intensity profiles (red) averaged from $>5$ single CAB-FITC-containing (b) or $\alpha$ ChT-FITC-containing (c) PDDA/PAA coacervate droplets, and associated standard deviations (gray) for samples prepared in the absence (top profile) or presence of 2, 4, 6 and $10 \mathrm{M}$ urea (lower profile). Arrow shows increasing urea concentrations. The distance $d$ was normalized to the size of the droplets $d_{0}$. 


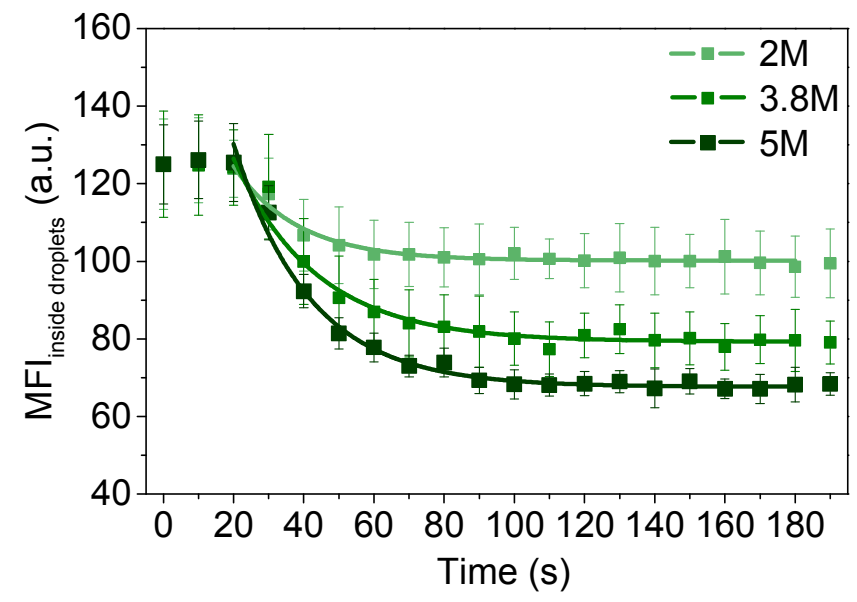

Figure S3: Plots showing time-dependent exponential decrease of the mean fluorescence intensity (MFI) inside individual coacervate micro-droplets after addition of various urea concentrations.

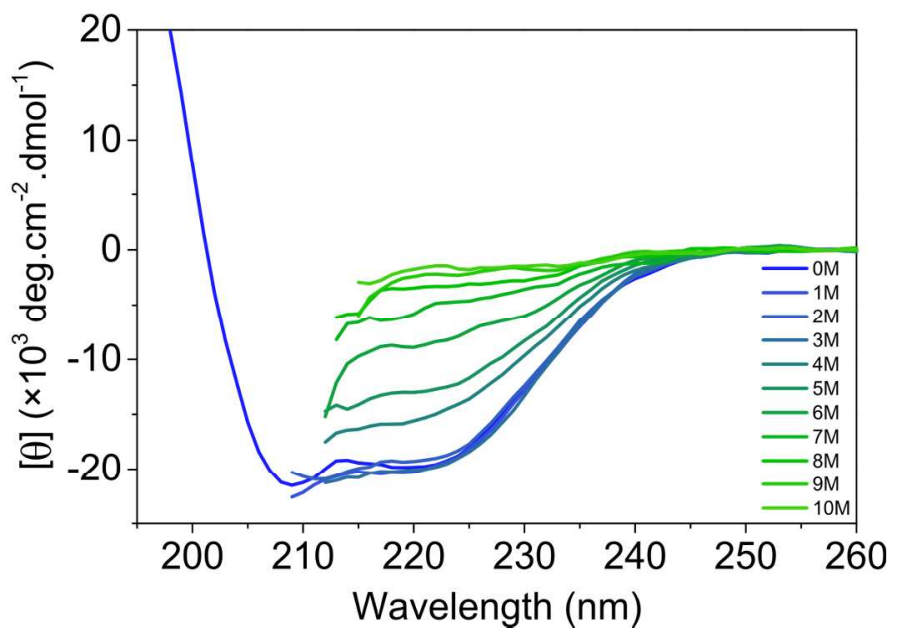

Figure S4. Far-UV SRCD spectra showing denaturation of BSA in buffers at increasing urea concentrations. 


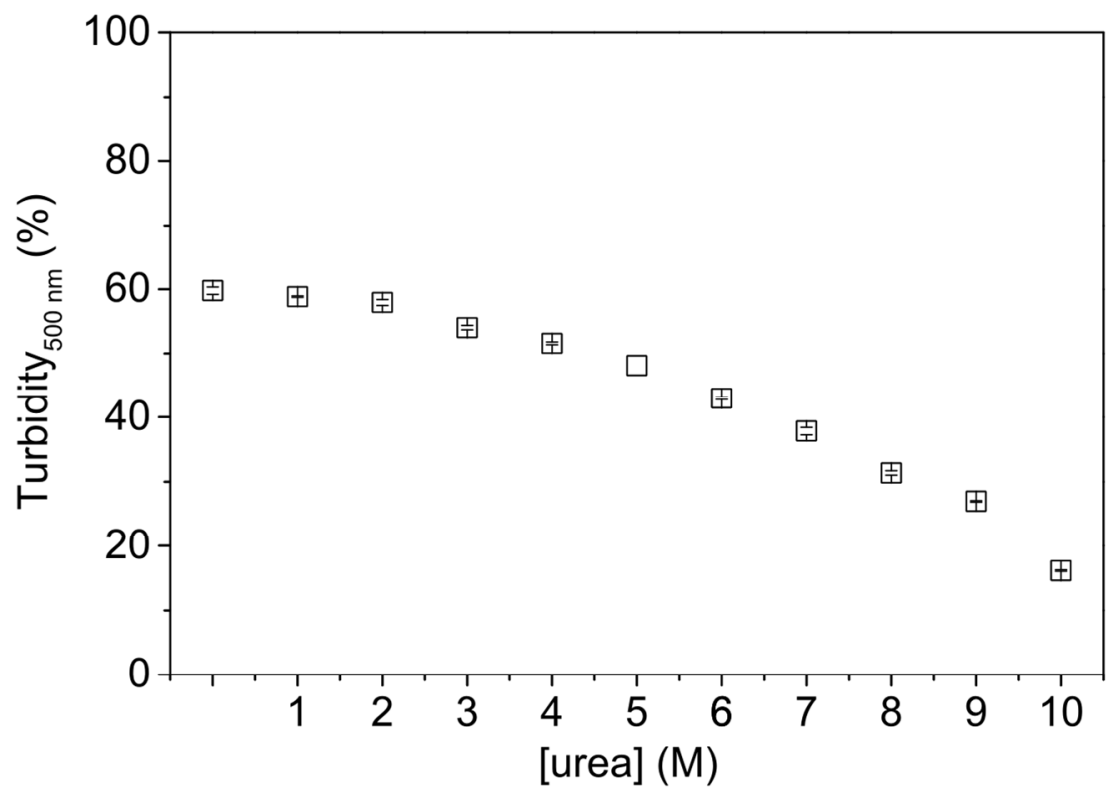

Figure S5. Plot of turbidity measured at $500 \mathrm{~nm}$ against urea concentration for a PDDA/PAA coacervate (final [monomer] $=25 \mathrm{mM}$; PDDA : PAA = 1:1). 

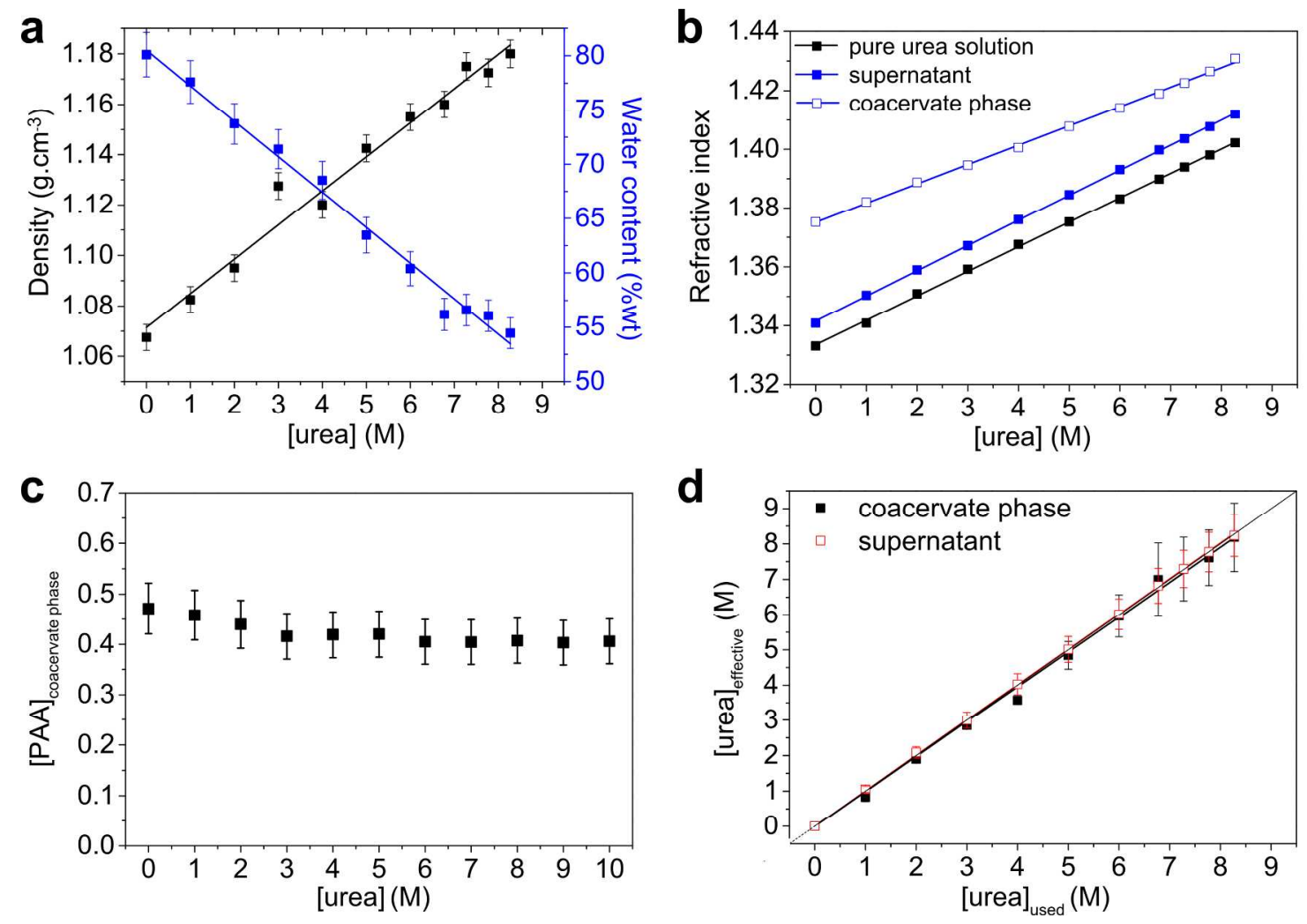

Figure S6. (a) Plots showing linear changes in density (black line) and water content (blue line) of PDDA/PAA coacervates $(\mathrm{pH}$ 8; final monomer concentration, $80 \mathrm{mM}$; monomer molar ratio = 1:1) prepared at urea concentrations between 0 and $8.5 \mathrm{M}$. A progressive decrease from 80 $w t \%$ (no urea) to $55 \mathrm{wt} \%$ at $8.5 \mathrm{M}$ urea, and corresponding increase in density from 1.07 to 1.18 $\mathrm{g} \mathrm{cm}^{3}$ are observed. (b) Plots showing urea-dependent linear increase in refractive index in aqueous urea solutions (control), coacervate bulk phase prepared as in (a), and supernatant. An increase from 1.38 to 1.43 is observed for the coacervate phase. (c) Plot showing approximately constant PAA concentration in PDDA/PAA coacervate bulk phases prepared as in (a) at increasing urea concentration. (d) Linear plots showing effective urea concentration in the coacervate bulk phase and supernatant as a function of the urea concentration used to prepare the samples. 


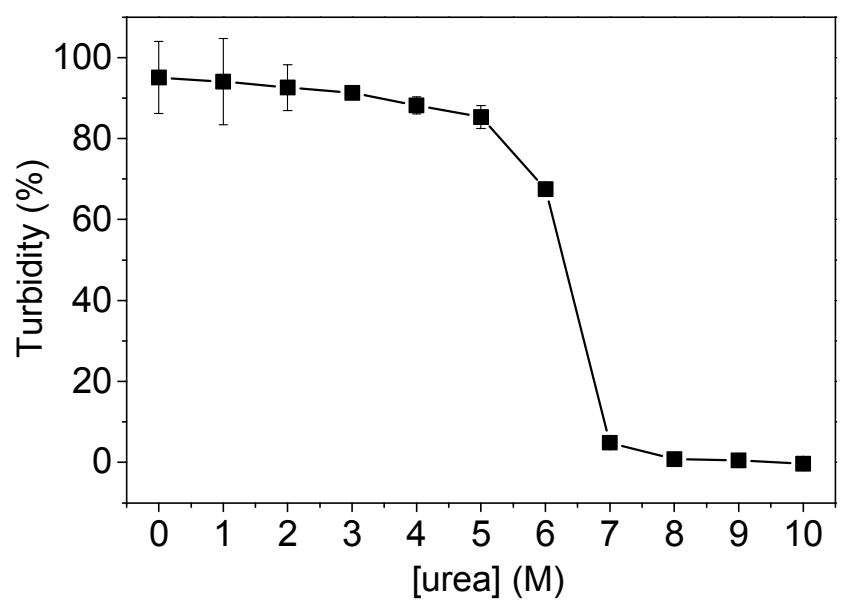

Figure S7. Plot showing decrease in turbidity for coacervates prepared from mixtures of PDDA and $\mathrm{BSA}\left([\mathrm{BSA}]=0.6 \mathrm{~g} \mathrm{~L}^{-1}=9 \mu \mathrm{M}, \mathrm{BSA}:\right.$ PDDA weight ratio $=10: 1$ ) with increasing urea concentration. The loss of turbidity is associated with disassembly of the coacervate phase due to unfolding of BSA and disassociation of the polymer/protein complex. 

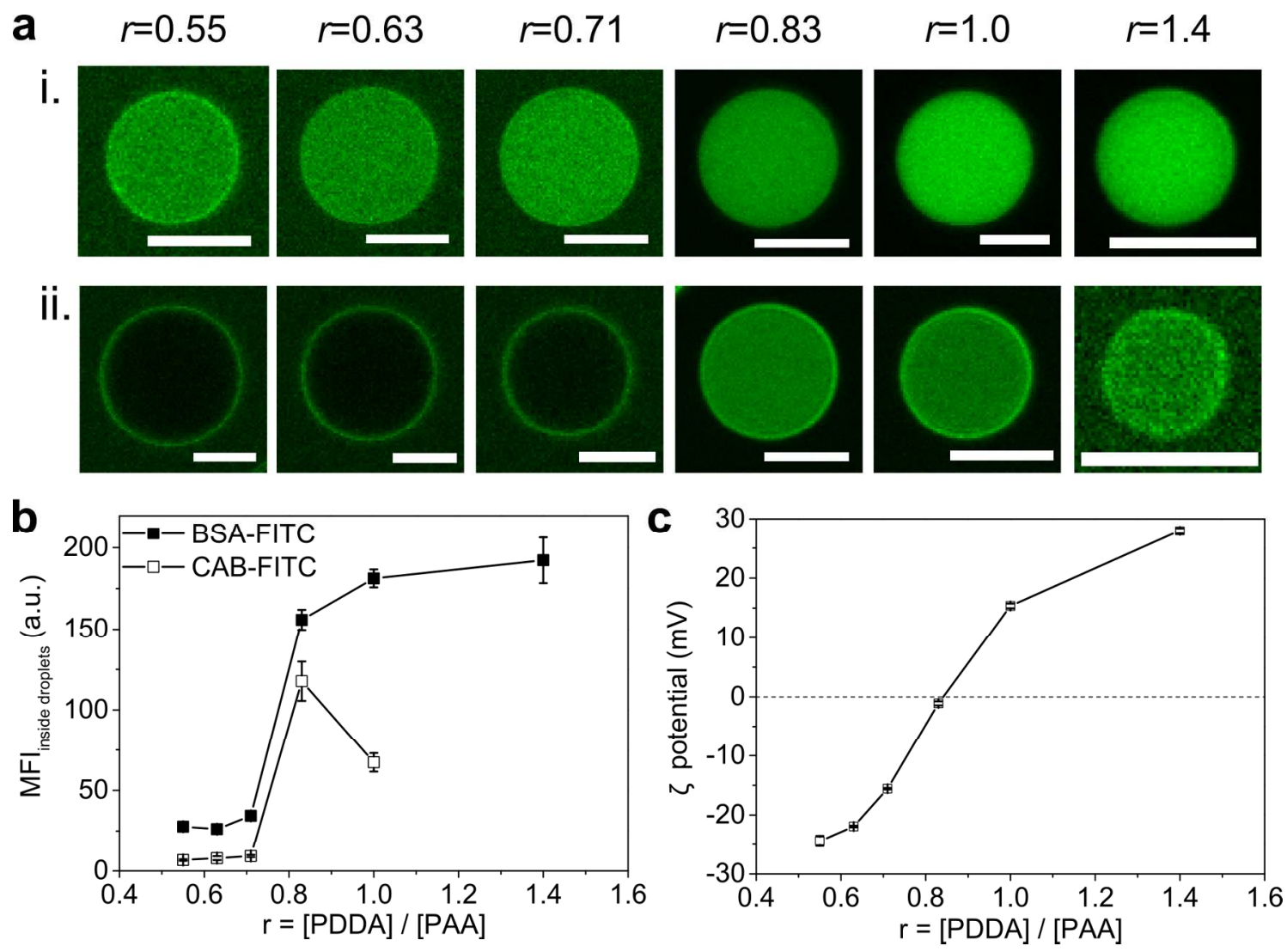

Figure S8. (a) Confocal fluorescence microscopy images of BSA-FITC (i) and CAB-FITC (ii) containing PDDA/PAA coacervate droplets prepared at different PDDA : PAA molar ratios $(r)$ ([PDDA monomer] = $25 \mathrm{mM}$ ); (negatively and positively charged droplets, $r \leq 0.83$ and $>0.83$ respectively); scale bars $5 \mu \mathrm{m}$. (b) Plot of mean fluorescence intensity (MFI) measured at the centre of BSA-FITC (black squares) or CAB-FITC (open squares) containing single coacervate droplets against $r$. (c) Plot of $\zeta$ potential of PDDA/PAA coacervate droplets against $r$.

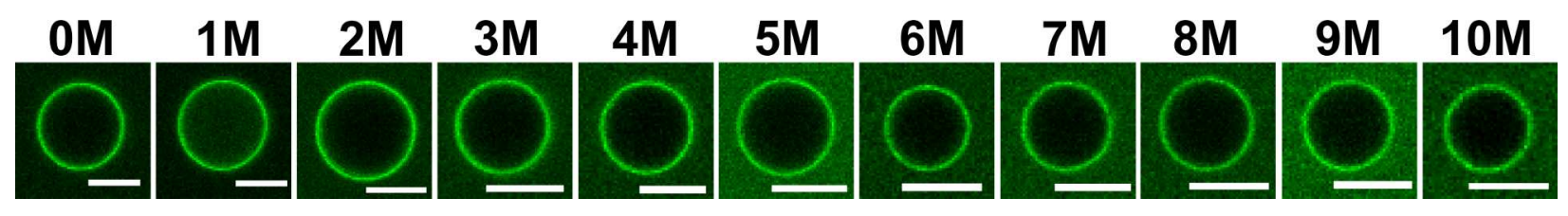

Figure S9. Confocal fluorescence microscopy images of negatively charged CAB-FITC-containing PDDA/PAA coacervate droplets (PDDA : PAA molar ratio $=0.71 ;[$ PDDA monomer $]=25 \mathrm{mM}$ ) prepared at urea concentrations between 0 and $10 \mathrm{M}$; scale bars $5 \mu \mathrm{m}$. 